\title{
A Pathway to Microdomain Alignment in Block Copolymer/Nanoparticle Thin Films under Electric Field
}

\author{
Joonwon Bae \\ Department of Applied Chemistry, Dongduk Women's University, Seoul 136-714, Korea \\ E-mail: joonwonbae@gmail.com \\ Received February 24, 2014, Accepted May 12, 2014
}

\begin{abstract}
The control over microstructure in block copolymer thin films using external electric fields has become an interesting research topic. In this article, the effect of nanoparticle on the microdomain alignments in block copolymer (polystyrene- $b$-poly(2-vinylpyridine)/nanoparticle ( $\mathrm{Au}$ ) thin films under electric fields has been examined with transmission electron microscopy. The homogeneous dispersion of Au nanoparticles into the block copolymer matrix was achieved by surface modification of nanoparticles with compatible ligands. Compared with the phenomenon seen in the pristine block copolymer thin films, a peculiar alignment behavior was observed in the block copolymer/nanoparticle hybrid thin films under electric fields. In addition, the different pathways observed in the pristine and nanoparticle incorporated block copolymer thin films were also monitored as a function of exposure time. This work can provide the fundamental information for understanding microdomain alignment in block copolymer/nanoparticle thin films under external electric fields.
\end{abstract}

Key Words : Block copolymer, Nanoparticle, Microdomain alignment, Electric field

\section{Introduction}

Block copolymers (BCPs) are fascinating materials composed of two or more polymer chains covalently bonded together at one end. BCPs have become more important both scientifically and technologically, because they possess elegant structures in tens of nanometers due to microphase separation behavior resulting from the strong segregation between constituent block. ${ }^{1}$ Depending on the composition of the constituent block chains, the microdomain shape can be either, spherical, cylindrical, gyroid, or lamellar. ${ }^{2}$ Recently, the spontaneous formation of nanostructures via the molecular self-assembly of block copolymers (BCPs) has attracted increasing scientific interest due to the growing number of applications. $^{3,4}$

On the other hand, owing to the recent interest toward functional materials, the blending of nanoparticles (NPs) and BCPs provides a novel means of creating hybrids that integrate the desirable features of each component. ${ }^{5,6}$ The properties of such hybrid materials depend on the physical properties and chemical nature of the dispersed and continuous phases. ${ }^{7,8}$ The use of these emerging composite materials requires understanding of profound structural, chemical, and physical properties of BCP/NP hybrids caused by the introduction of NPs into BCP matrix. ${ }^{9-14}$ Theoretical approaches predicted that interesting hierarchically ordered structures would be generated due to synergistic interactions between NPs and BCPs. ${ }^{10}$ This expectation was demonstrated experimentally by a couple of research groups. ${ }^{11-13}$ The locations of introduced NPs can be controlled microscopically through surface chemistry of NPs. ${ }^{13,14}$

Although numerous theoretical and experimental investigations on the microstructures and behaviors of $\mathrm{BCP} / \mathrm{NP}$ hybrids have been reported, there have been few systematic studies dealing with the interaction of $\mathrm{BCP} / \mathrm{NP}$ composites with an external electric fields. On the contrary, the interaction of an electric field with a dielectric material is well understood, since Swan first investigated the influence of electric fields on the surface of polymers or viscous liquids. ${ }^{15}$ For $\mathrm{BCPs}$, electric fields have been proven to be an effective mean to control the microdomain orientation in BCP thin films. ${ }^{16-26}$ The driving force for these processes is the orientation-dependent polarization in a material having dielectric interface. ${ }^{16}$ The electric field aligns interfaces separating two dielectric bodies parallel to the electric vector, thus minimizing the moment of electrostatic forces exerted onto the interface. ${ }^{17}$

There have been numerous previous studies regarding the microdomain alignments in block copolymer thin films under an electric field. ${ }^{16-26}$ Amundson et al. have initiated the interesting series of research works. ${ }^{16}$ Russell et al. have studied the effects of experimental parameters on the BCP microdomain orientation in the electric field. ${ }^{19}$ Zvelindovsky et al. have performed a profound theoretical investigation for understanding the phenomenon. ${ }^{25-26}$ Böker et al. have examined the fundamentals for the movements of microstructure in BCP under an electric field. ${ }^{20-22}$

In spite of the previous research effort, however, limited information regarding structures and behaviors of BCP/NP under electric fields have been available. ${ }^{27-29}$ Böker's group have reported the large-scale assembly of NPs in BCP templates $^{27,28}$ and the theoretical examination for dynamic behavior of BCP/NP microstructure under electric fields. ${ }^{29}$ Judging from those facts, it is worthwhile to observe directly the unique microphase behavior of $\mathrm{BCP} / \mathrm{NP}$ thin films under external electric fields to facilitate the understanding of the chemical and physical properties of those interesting hybrid 
materials. Therefore, in this study, the effect of NP on the microdomain alignment in BCP (Polystyrene- $b$-Poly(2-vinylpyridine)/NP thin films under electric fields was investigated with transmission electron microscope, compared with the microdomain alignment seen in the pristine BCP films. In addition, the different pathways observed in the pristine and nanoparticle incorporated block copolymer thin films were also monitored as a function of exposure time. In this study, it might be an additional advantage to observe and discuss the subtle interplay between a physical mean (electric field) and an interfacial interaction (ligand on NP and polymer matrix) directly.

\section{Experimental}

Materials. Poly(styrene)- $b$-poly(2-vinylpyridine) (PS- $b$ P2VP (Lamellar), Number average molecular weight : $\mathrm{M}_{\mathrm{n}}$, $\left.\mathrm{PS}=25 \mathrm{~kg} / \mathrm{mol}, \mathrm{P} 2 \mathrm{VP}=23 \mathrm{~kg} / \mathrm{mol}, \mathrm{M}_{\mathrm{w}} / \mathrm{M}_{\mathrm{n}}=1.08\right)$ was purchased from Polymer Source (Quebec, Canada) and used without further purification. Solvents, tetrahydrofuran (THF) was dried by a common method. Gold NPs were prepared by established procedures. ${ }^{30-32}$ For example, polystyrene-functionalized $\mathrm{Au}$ NPs ( $\mathrm{Au}(\mathrm{PS}))$, with an average gold diameter of $4 \mathrm{~nm}$, were prepared according to the Brust-Schiffrin method, by reduction of hydrogen tetrachloroaurate with sodium borohydride using polystyrenethiol as ligand. ${ }^{30}$ The molecular weight of the PS ligand was $1 \mathrm{~K}$, and the density of the $\mathrm{Au}(\mathrm{PS})$ was $19.3 \mathrm{~g} / \mathrm{cm}^{3}$.

Preparation of BCP/NP Hybrid Thin Films. Poly(styrene)- $b$-poly(2-vinylpyridine) (PS- $b$-P2VP) was dissolved in tetrahydrofuran (THF) at room temperature. THF is a good solvent for both PS and P2VP. Then an amount (1 vol \%) of Au NPs were added to the solution with a mild stirring. BCP/NP hybrid thin film was spin-coated onto silicon wafer at $2000 \mathrm{rpm}$ for $60 \mathrm{~s}$ from BCP/NP mixture solution. Then the hybrid thin films were dried under ambient conditions. Subsequently, the dried films were thermally annealed at $170{ }^{\circ} \mathrm{C}$, well-above the glass transition temperature of both blocks for at least $24 \mathrm{~h}$ before exposure to an electric field.

Application of Electric Field. A schematic diagram describing the apparatus in which the electric field was applied is shown in Figure 1. A silicon wafer, coated with a hybrid thin film, served as one electrode, and a soda-lime glass

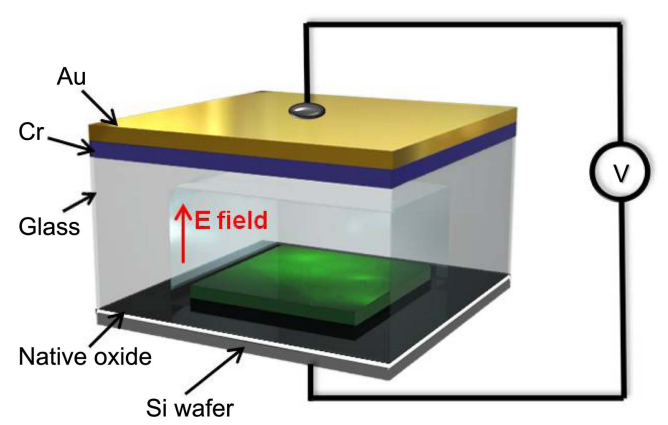

Figure 1. Schematic diagram of experimental apparatus. coated with a thin layer of gold $(20 \mathrm{~nm})$ and chromium as adhesion promoter $(5 \mathrm{~nm})$ was the second electrode. A rectangular well was etched in the glass slide by immersion in buffered HF using a poly(methyl methacrylate) mask. In this geometry, the dominant gradient in the electric field occurs across the air/nanocomposite film interface in Figure 1. A voltage was applied, and the assembly was heated for 6$24 \mathrm{~h}$ at $170^{\circ} \mathrm{C}$, well above the glass transition temperature of two blocks. Then the assembly was quenched to room temperature to freeze the pattern. After each experiment, the upper glass electrode was removed mechanically.

Instrumentations. Transmission electron microscopy (TEM) experiments were performed with a JEOL 200CX TEM operated at an accelerating voltage of $200 \mathrm{kV}$. First, the thin $\mathrm{BCP} / \mathrm{NP}$ films were spin coated onto a silicon wafer having a $250 \mathrm{~nm}$ thick silicon oxide layer. The oxidized silicon layer was etched with a $5 \%$ hydrofluoric acid (HF) aqueous solution. The separated polymer thin film was retrieved with a copper grid and examined by TEM. To prepare cross-sectional TEM specimens, a thin layer of carbon was evaporated onto the film surface before embedding in epoxy resin to prevent the diffusion of the epoxy resin into hybrid thin film. The thin film was then embedded in an epoxy resin and cured at $60{ }^{\circ} \mathrm{C}$ for $12 \mathrm{~h}$. The films were removed from the substrate by dipping into liquid $\mathrm{N}_{2}$. Ultrathin sections $(60 \mathrm{~nm})$ were collected at room temperature using a Leica Ultracut Microtome, equipped with a diamond knife. The thin sections were exposed to iodine vapor for $30 \mathrm{~min}$ to enhance the contrast.

\section{Results and Discussion}

To ensure the uniformity in properties, the confirmation of the homogeneous dispersion of Au NPs into the PS- $b$-P2VP matrix is critical. It is one of the most important prerequisites to examine the effect of NP on the microdomain alignments in BCP/NP thin films under electric fields. In general, the uniform distribution of NPs into the matrix has been achieved by tailoring surface functional groups to improve the compatibility between NPs and polymer., ${ }^{6,13,33}$ Figure 2(a) shows a TEM image of a PS- $b-\mathrm{P} 2 \mathrm{VP} / \mathrm{Au}(\mathrm{PS})$ hybrid thin film with an average thickness of approximately $50 \mathrm{~nm}$ containing 1 $\mathrm{vol} \% \mathrm{NPs}$. It is clear that the uniform dispersion of NPs into BCP is achieved. In this case, the enthalphic advantage for dispersing the particles is zero or small due to favorable interactions between the ligands and host polymer. ${ }^{33}$ In addition, the NPs are considerably small so that configurational entropic losses of polymer to incorporate the NPs are very small. ${ }^{34}$

Additional TEM experiment was performed to determine whether a coagulation/migration of the Au NPs happened inside the thin film. It is important to avoid such a nonuniform distribution of NPs, because it would distort the response of the BCN/NP hybrid thin film to an applied electric field. Figure 2(b) illustrates a cross-sectional TEM image of PS- $b$ $\mathrm{P} 2 \mathrm{VP} / \mathrm{Au}(\mathrm{PS})$ thin film with an average thickness of approximately $300 \mathrm{~nm}$ containing $1 \mathrm{vol} \% \mathrm{NPs}$. The absence of migration/segregation of the NPs to the film/air interface (white arrow) is clearly seen. Regardless of the viewing angle, 


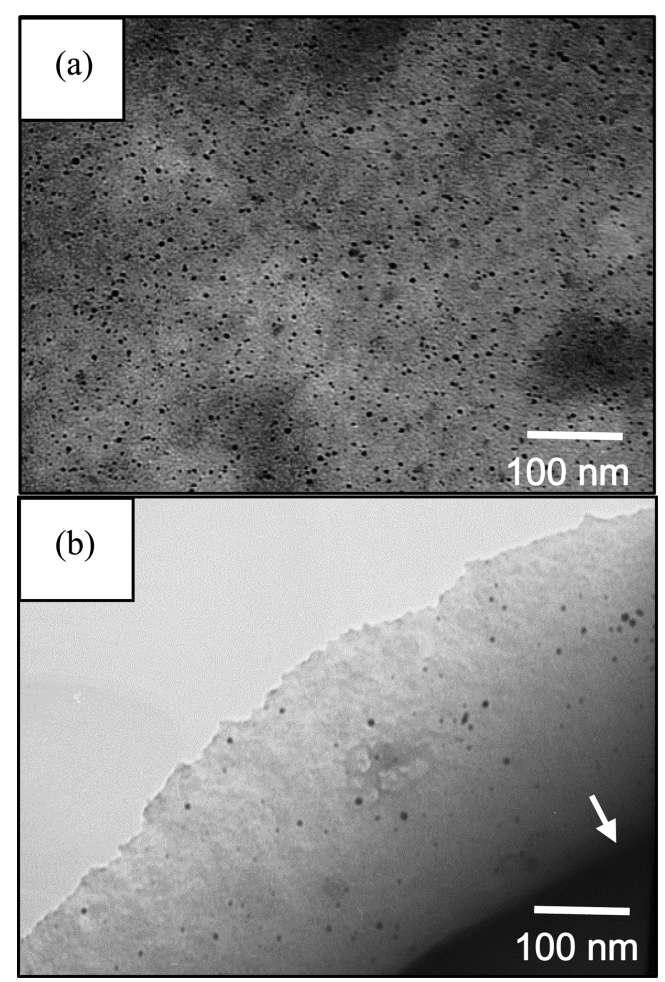

Figure 2. TEM image of (a) PS- $b-\mathrm{P} 2 \mathrm{VP} / \mathrm{Au}(\mathrm{PS})$ thin film with a thickness of $50 \mathrm{~nm}$ having $1 \mathrm{vol} \%$ nanoparticles and (b) crosssectional TEM image of PS- $b$-P2 VP/Au(PS) thin film with a thickness of $300 \mathrm{~nm}$ having $1 \mathrm{vol} \%$ nanoparticles. The white arrow indicates the film/air interface.

the NPs appear to be uniformly dispersed throughout the film before thermal annealing.

Subsequently, it was proceeded to observe the spatial locations of $\mathrm{Au}(\mathrm{PS}) \mathrm{NPs}$ inside PS- $b$-P2VP matrix after thermal annealing $\left(170{ }^{\circ} \mathrm{C} 48 \mathrm{~h}\right)$ to obtain an equilibrium microphase separated morphology. Figure 3 exhibits the cross-sectional TEM image of thermally annealed PS- $b-\mathrm{P} 2 \mathrm{VP} / \mathrm{Au}(\mathrm{PS})$ (1 vol \%) thin film after staining. To enhance contrast, TEM specimens were exposed to iodine vapor for a short time. Figure 3 indicates that Au(PS) NPs were exclusively located within PS domains. As the surface of Au NPs is entirely covered with PS ligands, the Au(PS) NPs are dominantly located in the center of PS microdomains. It is also clear that coagulation of NPs is not remarkable throughout the film. It is obvious that no migration/segregation happened during thermal annealing. Without external forces or fields, the coagulation or segregation of $\mathrm{Au}(\mathrm{PS}) \mathrm{NPs}$ is not energetically advantageous, because the ligand/matrix interaction is ideal for this system. Judging from Figure 3, it can be concluded that homogeneous dispersion as well as control over spatial location is achievable. ${ }^{34}$

An extensive TEM analysis was conducted to monitor the characteristic pathways to microdomain alignments in the pristine and NPs incorporated BCP thin films. Control experiment has been performed with a pristine PS- $b$-P2VP under an electric field $(15 \mathrm{~V} / \mathrm{mm})$. Figure 4 presents TEM images for a thermally annealed $\left(170^{\circ} \mathrm{C} 48 \mathrm{~h}\right)$ PS- $b$-P2VP

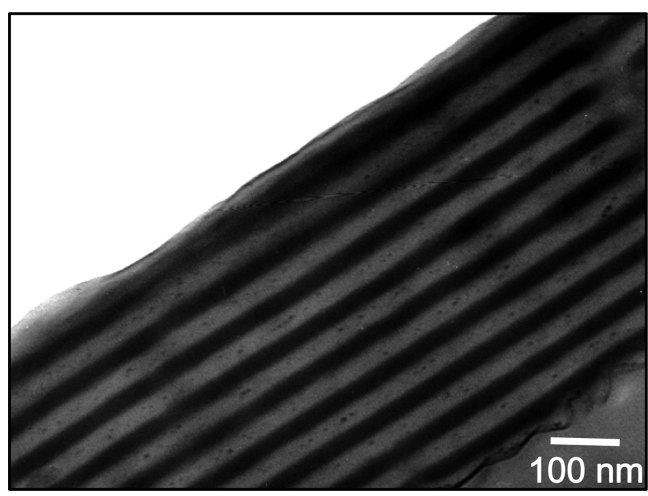

Figure 3. Cross-sectional TEM image of thermally annealed (170 ${ }^{\circ} \mathrm{C} 48$ h) PS- $b$-P2VP/Au(PS) thin film having 1 vol \% nanoparticles after staining with iodine.

copolymer thin film before (a) and after (b-d) exposure to an electric field- $15 \mathrm{~V} / \mu \mathrm{m} 170{ }^{\circ} \mathrm{C}$ as a function of exposure time. Figure 4(a) represents a typical equilibrium microphase separated morphology of PS- $b$-P2VP copolymer thin film. Because of the preferential wetting of P2VP block to a silicon wafer due to relatively higher surface energy, P2VP layer migrates to the bottom side of copolymer thin film during thermal annealing. It is clear that lamellar microdomains are aligned parallel to the substrate. On the other hand, Figure 4(b-d) show the microdomains oriented normal to the surface, adapted to the direction of an applied electric field (yellow arrow). When an applied electric field exceeds a critical value to overcome interfacial interaction, the surface/ interface becomes unstable. The electric field aligns interfaces separating two dielectric blocks parallel to the electric field vector. This leads to the formation of arrays of microdomains normal to the surface to minimize the moment of electrostatic forces exerted onto the interface between two blocks. It is notable that the vertical alignment does not extend completely to the interfaces, because the surface field effect exists at polymer/substrate interface (white arrow). On the contrary, in the center of the film, the effect of the substrate dissipates, so that microdomains oriented perpendicular to the substrate.

It is also discernible that the alignments by rotation started locally after $3 \mathrm{~h}$ exposure and then extended to the overall range in the middle of PS- $b$-P2VP thin film under an electric field. In general, the total pressure induced at the microdomain interfaces is given by Eq. (1), where $P_{e l}$ is the electrostatic pressure, $P_{l a p}$ is the Laplace pressure, and the $P_{d i s}$ disjoining force is generated by Van der Waals interaction. ${ }^{33}$

$$
P=P_{0}+P_{e l}(h)+P_{l a p}(h)+P_{d i s}(h)
$$

The electrostatic and Laplace force are common, but the disjoining pressure is strongly dependent on the presence of Van der Waals and surface interactions. For pristine block copolymer films, two major forces exist at interface-an electrostatic force applied by the electric field and a torque attributed to the rotation of the microdomain by electrostatic force. That is, the microdomain interfaces are macroscopically in the equilibrium state, thus microdomains rotate under the 
electric field without crossover in the microdomain. The contribution of electrostatic force is much higher than that of other force, the alignments are supposed to start upon exposure to an electric field. But, it took more time utill the alignments

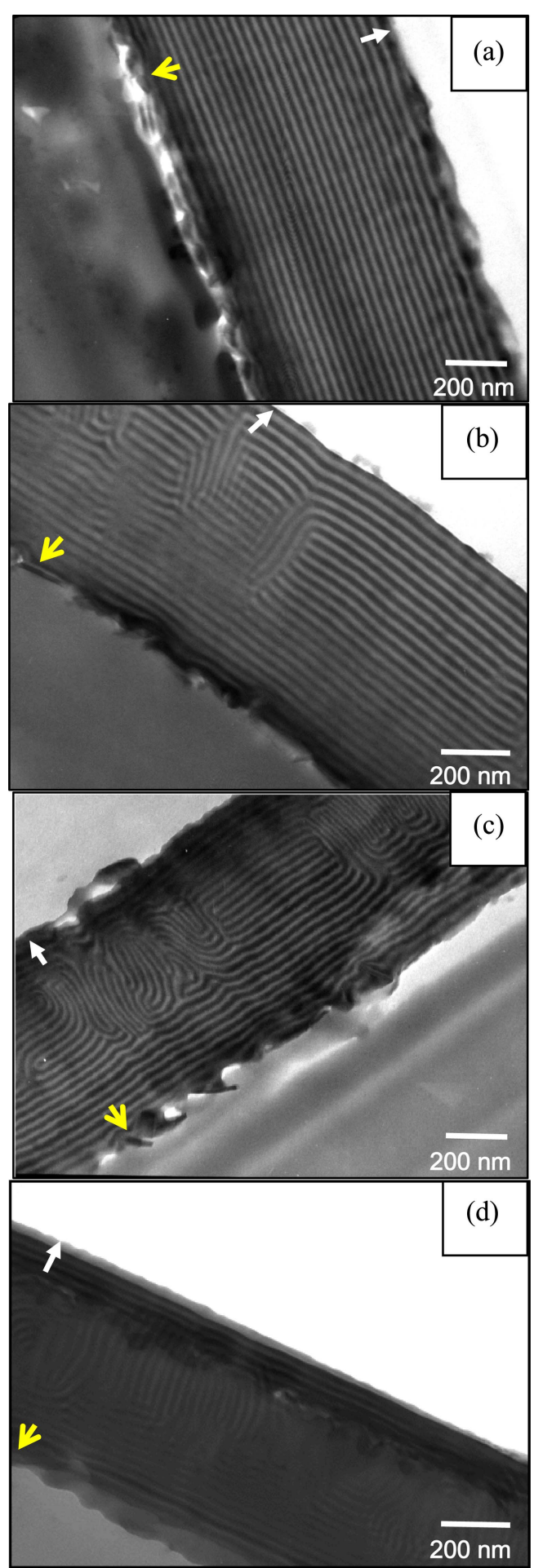

Figure 4. Cross-sectional TEM images of pristine PS- $b-\mathrm{P} 2 \mathrm{VP}$ thin film (a) thermally annealed $\left(170{ }^{\circ} \mathrm{C} 48 \mathrm{~h}\right)$ and exposed to an electric field $15 \mathrm{~V} / \mu \mathrm{m}$ at $170{ }^{\circ} \mathrm{C}$ for (b) $3 \mathrm{~h}$, (c) $6 \mathrm{~h}$, and (d) $12 \mathrm{~h}$. The images were taken after staining with iodine. The white and yellow arrows indicate the film/substrate and film/air interface, respectively. extend to the entire film.

To reveal the microdomain alignments in PS- $b$-P2VP/ $\mathrm{Au}(\mathrm{PS})(1.0 \mathrm{vol} \%)$ thin film under an electric field, crosssectional TEM images are shown in Figure 5. The image in Figure 5 verifies that the mixed microdomain orientation in $\mathrm{BCP} / \mathrm{NPs}$ thin film is predominant throughout the hybrid thin film. Parallel orientations to substrate were universal at the vicinity of substrate (yellow arrow) and thin film/air interface (white arrow). Random/mixed orientation is prevalent in the middle of hybrid thin films. Importantly, the aspect of microdomain orientation under an electric field is significantly different between PS- $b$-P2VP and PS- $b$-P2VP/ $\mathrm{Au}(\mathrm{PS})$.

This striking difference might be discussed in terms of two possible ways to microdomain orientations. One is a simple rotation of microdomain grains. This process is energetically

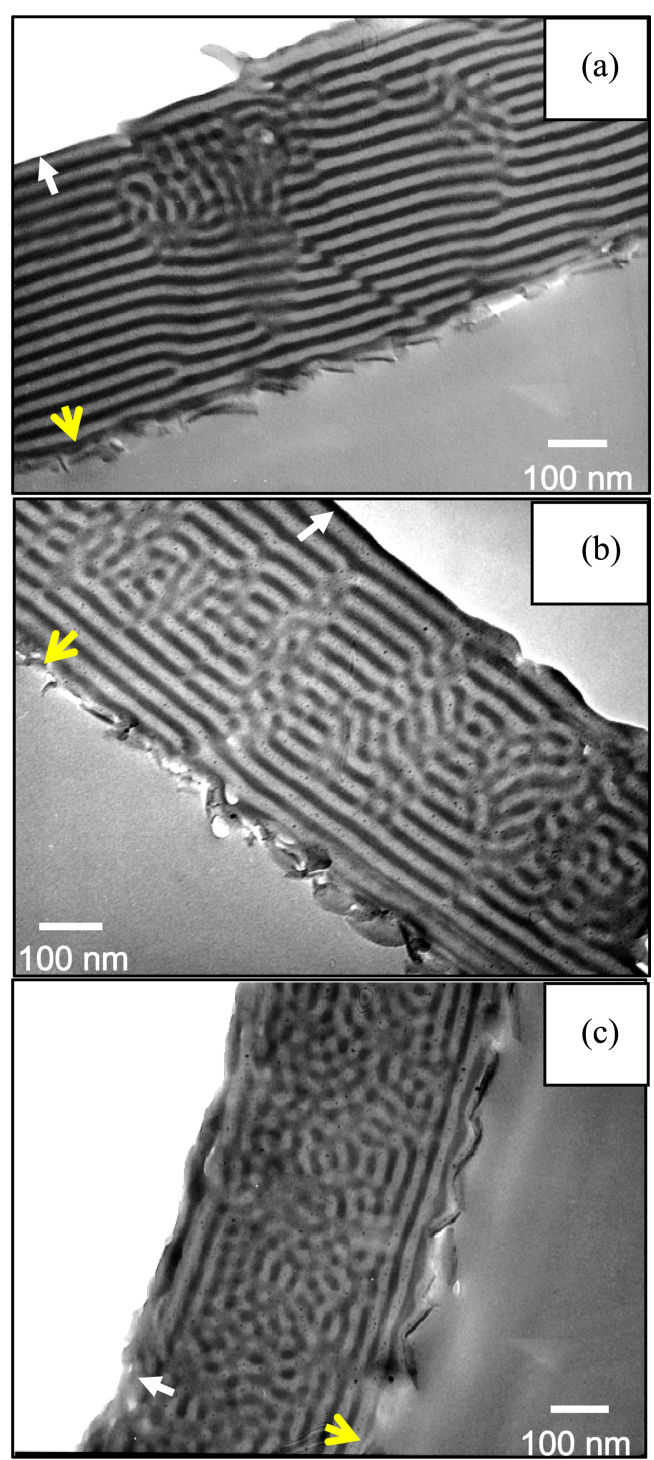

Figure 5. Cross-sectional TEM images of PS- $b-\mathrm{P} 2 \mathrm{VP} / \mathrm{Au}(\mathrm{PS})$ NPs thin film having $1 \mathrm{vol} \%$ nanoparticles exposed to an electric field $15 \mathrm{~V} / \mu \mathrm{m}$ at $170{ }^{\circ} \mathrm{C}$ for (a) $3 \mathrm{~h}$, (b) $6 \mathrm{~h}$, and (c) $12 \mathrm{~h}$. The images were taken after staining with iodine. The white and yellow arrows indicate the film/substrate and film/air interface, respectively. 
nonfavorable, because a high energy barrier is associated with a complete rotation. A more feasible route is through an undulation of the microdomains. Local variations in NPs population density in microdomain could generate microscale imbalances in electrostatic force inside microdomains, promoting interfacial fluctuations. Therefore, the possibility for interfacial undulation in BCP/NPs thin film increases dramatically. This also means that the NPs played a role of defect to microdomain orientations. The presence of defects results in the generation of crossed microdomains. ${ }^{35}$

On the other hand, the dependence of microdomain alignments in BCP/NP thin film on exposure time is macroscopically similar to that seen in the pristine BCP thin film (Figure 4). This is because the introduction of NPs into BCP did not significantly change the overall dielectric constant of one block.

The unprecedented microdomain alignment in Figure 5 compared with Figure 4 can be understood in terms of strong electric field and microphase mixing effect induced by the presence of NPs. In any case, the TEM image in Figure 5 is conspicuous, which is not accessible by any other means. It can be inferred that the combination of a physical meanelectric field and favorable interfacial interaction between ligand on NP and BCP matrix generated the interesting morphology. Strictly speaking, TEM is the most effective way of delivering eye-friendly information to the researchers. Thus we have investigated numerous samples using normal and cross-sectional TEM study. However, the overall and longrange phenomena must be examined by X-ray analysis. This work can be a separate topic for future publication.

\section{Conclusion}

The effect of Au nanoparticles on microdomain alignments in block copolymer/nanoparticle hybrid thin films under electric fields was investigated systematically using crosssectional TEM experiments. Homogeneous and controlled placement of nanoparticles inside copolymer matrix was achieved by surface engineering of nanoparticles. The microdomain alignment behavior of block copolymer/nanoparticle thin films is significantly different from that of pristine block copolymer films. The presence of nanoparticles induces random/crossed microdomain orientations under electric fields. However, it has been common phenomenon that the microdomain alignments commence locally and extend throughout the thin films. This work is meaningful for understanding the profound properties of emerging block copolymer/nanoparticle hybrid thin films for application in polymeric and carbon nanomaterials.

Acknowledgments. This work was supported by Dongduk Women's University grant.

\section{References}

1. Hamley, I. W. Introduction to Block Copolymers. in Developments in Block Copolymer Science and Technology; John Wiley and
Sons: London, UK, 2004.

2. Hamley, I. The Physics of Block Copolymers; Oxford University Press: Oxford, UK, 1998.

3. Park, C.; Yoon, J. S.; Thomas, E. L. Polymer 2003, 44, 6725.

4. Park, M.; Harrison, C.; Chaikin, P. M.; Register, R. A.; Adamson, H. Science 1997, 276, 1401.

5. Black, C. T.; Murray, C. B.; Sandstrom, R. L.; Sun, S. H. Science 2000, 290, 1131.

6. Kim, B. J.; Fredrickson, G. H.; Kramer, E. J. Macromolecules 2008, 41,436

7. Tseng, R. J.; Tsai, C. L.; Ma, L. P.; Ouyang, J. Y. Nature Nanotech. 2006, 1,72 .

8. Konstantatos, G.; Howard, I.; Fischer, A.; Hoogland, S.; Clifford, J.; Klem, E.; Levina, L.; Sargent, E. H. Nature 2006, 442, 180.

9. Zeng, Q. H.; Yu, A. B.; Lu, G. Q. Prog. Polym. Sci. 2008, 33, 191.

10. Ginzburg, V. V.; Qiu, F.; Paniconi, M.; Peng, G.; Jasnow, D.; Balazs, A. C. Phys. Rev. Lett. 1999, 82, 4026.

11. Yan, L. T.; Popp, N.; Ghosh, S. K.; Böker, A. ACS Nano 2010, 4, 913.

12. Warren, S. C.; Messina, L. C.; Slaughter, L. S.; Kamperman, M.; Zhou, Q.; Gruner, S. M.; DiSalvo, F. J.; Wiesner, U. Science 2008, $320,1748$.

13. Chiu, J. J.; Kim, B. J.; Kramer, E. J.; Pine, D. J. J. Am. Chem. Soc. 2005, 127, 5036.

14. Deshmukh, R. D.; Liu, Y.; Composto, R. J. Nano Lett. 2007, 7, 3662.

15. Swan, J. W. Proc. R. Soc. London 1897, 62, 38.

16. Amundson, K.; Helfand, E.; Quan, X.; Smith, S. D. Macromolecules 1993, 26, 2698.

17. Böltau, M.; Walheim, S.; Mlynek, J.; Krausch, G.; Steiner, U. Nature 1998, 391, 877

18. Gurovich, E. Macromolecules 1994, 27, 7339.

19. Xu, T.; Zhu, Y.; Gido, S. P.; Russell, T. P. Macromolecules 2004, $37,2625$.

20. Liedel, C.; Pester, C. W.; Ruppel, M.; Urban, V. S.; Böker, A. Macromol. Chem. Phys. 2012, 213, 259.

21. Ruppel, M.; Pester, C. W.; Langner, K. M.; Sevink, G. J. A.; Schoberth, H. G.; Schmidt, K.; Urban, V. S.; Mays, J. W.; Böker, A. ACS Nano 2013, 7, 3854.

22. Schmidt, K.; Schoberth, H. G.; Ruppel, M.; Zettl, H.; Hänsel, H.; Weiss, T. M.; Urban, V. S.; Krausch, G.; Böker, A. Nature Mater. 2007, 7, 142.

23. Yan, L. T.; Xie, X. M. Prog. Polym. Sci. 2013, 38, 369.

24. Goldberg-Oppenheimer, P.; Kabra, D.; Vignolini, S.; Hüttner, S.; Sommer, M.; Neumann, K.; Thelakkat, M.; Steiner, U. Chem. Mater. 2013, 25, 1063.

25. Ly, D. Q.; Pinna, M.; Honda, T.; Kawakatsu, T.; Zvelindovsky, A. V. M. J. Chem. Phys. 2013, 138, 074904.

26. Pinna, M.; Schreier, L.; Zvelindovsky, A. V. M. Soft Matter 2009, 5,970 .

27. Yan, L. T.; Schoberth, H. G.; Böker, A. Macromol. Chem. Phys. 2009, 210, 1003.

28. Liedel, C.; Pester, C. W.; Ruppel, M.; Lewin, C.; Pavan, M. J.; Urban, V. S.; Shenhar, R.; Bösecke, P.; Böker, A. ACS Macro Lett. 2013, 2, 53.

29. Yan, L. T.; Schoberth, H. G.; Böker, A. Soft Matter 2010, 6, 5956.

30. Brust, M.; Walker, M.; Betell, D.; Schiffrin, D. J.; Whyman, R. J. J. Chem. Soc. Chem. Commun. 1994, 801.

31. Daniel, M. C.; Astruc, D. Chem. Rev. 2004, 104, 293.

32. Jackson, A. M.; Myerson, J. W.; Stellacci, F. Science 2007, 315, 358.

33. Bae, J.; Glogowski, E.; Gupta, S.; Chen, W.; Russell, T. P. Macromolecules 2008, 41, 2722.

34. Gupta, S.; Zhang, Q.; Emrick, T.; Russell, T. P. Nano Lett. 2006, 6, 2066.

35. Olszowka, V.; Hund, M.; Kuntermann, V.; Scherdel, S.; Tsarkova, L. A.; Böker, A. ACS Nano 2009, 5, 1091. 